\title{
Desarrollo de un Modelo de Interlocución para el Sistema de Salud basado en el Caso de la Guajira Colombiana
}

\author{
Developing an interlocution model for the health system based on \\ studying the Colombian Guajira
}

Claudia Puerta-Silva y Robert VH Dover

Instituto de Estudios Regionales INER y Departamento de Antropología, Universidad de Antioquia, Colombia. cpuerta@iner.udea.edu.co; rdover@une.net.co

Recibido 15 Diciembre 2006/Enviado para Modificación 30 Abril 2007/Aceptado 12 Julio 2007

\section{RESUMEN}

Objetivos Este artículo busca mostrar el desarrollo de un Modelo de Interlocución sobre el desempeño local del sistema de salud en La Guajira, entre 2005 y 2007, con el fin de generar condiciones para la participación social y el mejor desempeño del sistema conforme a las condiciones, necesidades y expectativas locales.

Métodos El desarrollo del Modelo se hizo mediante técnicas etnográficas, en la primera y segunda fase de la investigación, y técnicas participativas, en la tercera. La metodología buscaba recoger la información que permitiera escoger las estrategias de intervención para mejorar las habilidades y capacidades para la participación de los usuarios y que sugiriera la creación de espacios de confianza para la interlocución entre los actores y la negociación de acciones de mejoramiento para el sistema. Con base en la etnografía del sistema y del usuario, se diseñaron estrategias de intervención que incluyen materiales impresos pedagógicos y tres módulos en forma de talleres participativos. Se probó el Modelo en cuatro localidades de La Guajira, en donde al final se crearon Mesas de Trabajo en Salud con participación de usuarios y decisores institucionales.

Resultados Se constató que se puede lograr que los usuarios participen como ciudadanos informados y consumidores críticos, fortaleciendo sus habilidades de interlocución, ampliando sus conocimientos, generando léxicos y significados compartidos con los actores institucionales, y utilizando los dispositivos locales y redes sociales de participación.

Conclusión Es indispensable encontrar estrategias efectivas para motivar una mayor participación de las instituciones, especialmente de las Entidades Promotoras de Salud y Aseguradoras del Régimen Subsidiado.

Palabras Clave: Participación Comunitaria, planificación social, controles informales de la sociedad, calidad de la atención de salud, sistemas de salud (fuente: DeCS, BIREME). 


\section{ABSTRACT}

Objectives This article shows the development of an interlocution model regarding the health system's local performance in La Guajira from 2005 to 2007. It was aimed at producing conditions for social participation and improving the system's performance according to local conditions, needs and expectations.

Methods Such model was developed by using ethnographic techniques during the investigation's first and second phases and participative techniques during its third phase. The methodology sought to collect information leading to choosing intervention strategies for improving user-participation ability and capacity and creating spaces of trust for interlocution between the actors and negotiating action for improving the system. Intervention-strategies were based on system and user ethnography, including printed pedagogical material and three modules in the form of participative workshops. The model was tested in four localities in the Guajira, leading to working tables for health being created, encouraging participation by users and institutional decision-makers.

Results It was noted that users could participate as informed citizens and critical consumers, strengthening their interlocution abilities, broadening their knowledge, producing vocabulary and meaning shared with the institutional actors and using local mechanisms and social participation networks.

Conclusion Effective strategies must be found for motivating greater participation by institutions, especially health promoting entities and subsidised regime insurers.

Key Words: Consumer participation, social planning, social control informal, quality of health care, health systems (source: $M e S H, N L M$ ).

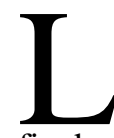

a investigación "Representación de las comunidades locales en el régimen subsidiado de salud" desarrolló e implementó el Modelo de fin de generar procesos para que los usuarios incidan real y efectivamente en el desempeño del sistema (1). Nuestra hipótesis principal es que las comunidades locales no están suficientemente representadas, primero porque el sistema no alcanza a responder a sus necesidades y expectativas, y segundo, porque los usuarios no saben o no les interesa participar. Otra hipótesis asume que para lograr el mejoramiento del sistema, es fundamental fortalecer las capacidades de los usuarios para que su participación informada y eficiente impacte realmente.

Con la etnografía buscamos definir estrategias adecuadas para aumentar conocimientos, mejorar habilidades y capacidades de participación, y crear espacios y mecanismos de interlocución sostenibles y efectivos. Escogimos La Guajira, pues consideramos que representa todos los retos posibles para el 
régimen de aseguramiento subsidiado: diversidad poblacional, bajos niveles de escolaridad y población mayoritaria en condiciones socioeconómicas vulnerables, dispersión demográfica y población flotante, condiciones geográficas y ambientales adversas, precaria infraestructura de servicios públicos, débil desarrollo institucional, proliferación de Instituciones Prestadoras de Servicios (IPS) y Administradoras (ARS), descentralización incompleta, alto ingreso de regalías por minería, y recaudo de impuestos casi nulo. Supusimos que un modelo desarrollado en este contexto tendría en cuenta las condiciones más extremas de este régimen de aseguramiento en salud para las poblaciones vulnerables.

El Modelo de Interlocución es un conjunto de procedimientos metodológicos que propician las condiciones adecuadas y los espacios de interlocución de confianza y no beligerantes, para la comunicación efectiva. En la Mesa de Trabajo en Salud, usuarios y agentes del sistema o institucionales (servidores públicos, decisores, guías educativos, promotores, atención al usuario, personal asistencial), identifican conjuntamente los problemas, factores y actores involucrados, para concertar acciones de mejoramiento.

Después de la reforma del sistema colombiano las metas de calidad y eficiencia tenían el fin de garantizar mayor competencia y opciones de servicios. Es en este contexto que la perspectiva del usuario y el fortalecimiento de medidas democráticas, resultan fundamentales en el propósito del mejoramiento de la salud, así como también, en la consolidación de los controles burocráticos, profesionales y administrativos, en la reducción del abuso del poder, la ineficiencia y la corrupción, y en el mejoramiento de la calidad (2-4).

La reforma colombiana introdujo y adoptó mecanismos de participación individual y colectiva. Como ciudadanos, los usuarios tienen derecho al acceso, equidad y calidad, y como consumidores tienen opciones, como el "retiro" y la libre escogencia (5). Pero para el ejercicio como ciudadano y consumidor, el afiliado al régimen subsidiado requiere de un conocimiento básico sobre derechos y deberes, sobre lo que puede esperar, y sobre cómo exigir calidad. Este conocimiento es una de las condiciones para que ellos tengan la misma capacidad de interlocución, el mismo léxico que los agentes institucionales y la disposición de concertar soluciones localmente.

La idea de la interlocución surge con el ánimo de traspasar modelos como el de la comunicación para el desarrollo, compuestos por un emisor, un mensaje y un receptor (6-8). En estos modelos, la retroalimentación del mensaje sólo 
sirve para corroborar el mensaje del emisor; el receptor no es un sujeto activo, sino un objeto. El Modelo de Interlocución busca equilibrar el poder de la comunicación, unificando conceptos y léxicos, y habilitando al supuesto "receptor" como interlocutor válido, pero también haciendo que la formulación de políticas esté informada por las necesidades comunitarias (6; 9-12). La interlocución apela aquí a una definición de comunicación amplia, según la cual los sujetos se influencian mutualmente y la comunicación misma está influenciada por el contexto (7-8). Por ello proponemos un Modelo que tiene en cuenta aspectos culturales y sociales de la interacción entre actores del sistema, y que busca equilibrar las relaciones de poder y conocimiento. Se trata de convertir la participación en un diálogo sostenible para la toma de decisiones, la planificación y el control. Ahora bien, la literatura consultada se concentra en el análisis de la participación y no se habla de los procesos comunicativos que ella encierra (2-3,10,12-14).

Queremos transformar al receptor en interlocutor mediante la implementación de este Modelo, y para ello, es necesario convertir al "paciente" o "afiliado", en un "usuario informado" y "consumidor crítico", conciente de lo que puede esperar del sistema y de cómo exigir satisfacción, es decir, convertirlo en un interlocutor válido y horizontal del agente institucional. Este artículo se concentra en la descripción del desarrollo del Modelo de Interlocución sin profundizar en las condiciones que consideramos estructuran el funcionamiento del sistema de salud y la participación ciudadana en Colombia, y particularmente en La Guajira.

\section{METODOLOGÍA}

La investigación se distribuyó en tres fases, en las cuales se aplicaron metodologías básicas y aplicadas de investigación social, con el fin de desarrollar el Modelo y probarlo en cuatro localidades. La investigación básica consistió en el análisis etnográfico y político, la revisión documental de las instituciones y la revisión bibliográfica sobre sistemas de salud y participación ciudadana. La aplicada, fundamentada en la etnografía, buscó probar la pertinencia de estrategias informativas y participativas para el fortalecimiento de la capacidad de los usuarios, pero también las posibilidades de la creación de espacios y mecanismos de interlocución.

Durante las dos primeras fases (octubre 2005 y febrero-mayo 2006), identificamos los factores locales que inciden en el desempeño del régimen subsidiado, y las condiciones existentes y necesarias para la participación. En 
la tercera fase (septiembre 2006), distribuimos el material educativo y realizamos talleres, para la creación de las Mesas de Trabajo en Salud.

Cuatro localidades de La Guajira -Riohacha, Maicao, Albania y Nazareth, corregimiento de Uribia-, fueron seleccionadas con base en criterios de representatividad descritos más adelante.

\section{Primera Fase}

El trabajo etnográfico, con entrevistas semi-estructuradas y colectivas, observación directa y análisis de documentos institucionales, logró: 1) diagnósticos institucionales del funcionamiento del sistema y caracterizaciones de los factores que inciden y determinan su desempeño, útil en la contextualización para identificar las posibilidades y limitaciones del sistema para su adecuación y planificación local, los perfiles epidemiológicos, las condiciones ambientales, las lógicas, interpretaciones de la normatividad y comportamientos de los agentes institucionales que influyen en la cobertura, el acceso, la eficiencia y la calidad de los servicios; 2) identificar espacios de participación, su funcionamiento, su promoción por las entidades y los objetivos; 3) caracterizar a las poblaciones beneficiarias y las vulnerables, sin ningún régimen de aseguramiento.

\section{Segunda Fase}

Partiendo de la primera clasificación de los usuarios y poblaciones afiliadas al régimen subsidiado 1 ) se identificaron sus características y su campo relacional con el sistema y 2) se diseñaron las estrategias de intervención para generar habilidades, capacidades, mecanismos y espacios de interlocución. Durante un trabajo etnográfico de tres meses, el equipo de investigación realizó entrevistas semi-estructuradas y colectivas, conversatorios, observación directa en los puntos de atención de usuarios en Secretarías de Salud, ARS e IPS, encuestas, y visitas a rancherías, barrios y corregimientos. Definimos primero las localidades y luego las comunidades con las que trabajaríamos. Para ello tomamos, principalmente como criterios de representatividad, los siguientes: la ubicación geográfica con relación a los centros urbanos y por lo tanto a las instituciones de salud; el tipo de población (estrato socio-económico, vocación económica, y auto-definición étnica); los niveles de escolaridad, de organización política, y de participación social y gestión comunitaria; los perfiles epidemiológicos y riesgos ambientales para la salud. Otros factores incidieron también en la escogencia de los casos y subcasos: el interés y voluntad expresados por las comunidades y sus líderes, y las posibilidades operativas (transporte, condiciones de seguridad, etc.). 
Definimos perfiles de usuarios, con base en la clasificación institucional de vulnerabilidad, pero especialmente, por su conocimiento y nivel de contacto con el sistema. Se entrevistaron usuarios con conocimiento básico (saben lo mínimo para acceder con el carné a servicios de salud) y usuarios-líderes (saben los mecanismos para resolver problemas básicos en el acceso a los servicios). Entre estos se buscó la representatividad de mujeres y hombres, jóvenes, adultos, indígenas, afro-descendientes, campesinos, inmigrantes, desplazados y población urbana.

Con todos estos datos identificamos los contextos condicionantes de la participación, y las características y capacidades de los usuarios, y diseñamos estrategias de intervención que tuvieron dos componentes: materiales educativos impresos y talleres, los cuales tenían como objetivo contribuir 1) al mejoramiento de las capacidades y habilidades de usuarios y agentes, y 2) a la sensibilización de los actores frente a la participación. Tanto en los materiales impresos como en los talleres buscamos mostrar las limitaciones y posibilidades del régimen subsidiado para responder a condiciones, necesidades y expectativas locales, y las ventajas de la organización y participación comunitaria en este sentido.

Se produjeron los siguientes materiales impresos: 1) Guía práctica de orientación al usuario del Régimen Subsidiado: Manual sobre la estructura, funcionamiento y normatividad del sistema, derechos y deberes, formas y espacios de participación y de protección. Dirigido a líderes con nivel de escolaridad y a los agentes institucionales de primer contacto. 2) Cartilla sobre el Régimen Subsidiado: Cuadernillo básico sobre el régimen subsidiado, deberes y derechos, formas y espacios de participación y protección, para usuarios con capacidades medias lectoescritoras. 3) Plegable: Informativo básico y rápido sobre el régimen subsidiado, para usuarios con capacidades básicas lectoescritoras. 4) Afiche: Diagrama de los actores y procesos del régimen subsidiado. Ilustra la trayectoria del usuario en el sistema. Dirigido a usuarios y agentes, para ser expuesto en las instituciones.

\section{Tercera Fase}

En la tercera fase realizamos los talleres participativos en los subcasos, distribuimos los materiales pedagógicos y se instalaron las Mesas de Trabajo en Salud. Estos talleres se dividieron en tres módulos, que tenían como propósito llevar a los actores del sistema local de salud a una Mesa de Trabajo en Salud, en la cual podrían concertar soluciones conjuntas y definir responsabilidades y plazos, en un ambiente propicio para la interlocución. El módulo 1 aporta herramientas para usar el sistema de salud y contribuir a su mejoramiento a los 
usuarios y líderes comunitarios; el módulo 2, busca fortalecer la participación y la organización comunitaria, sensibilizando a usuarios-líderes y a funcionarios y empleados de primer contacto de Secretarías de salud, IPS y ARS; el módulo 3, propicia la creación de la Mesa de Trabajo en Salud, como un espacio y mecanismo de interlocución y negociación, entre usuarios-líderes y decisores de las Secretarías de salud, IPS y ARS.

Durante septiembre de 2006, el equipo realizó las actividades en cada caso durante una semana. En todos los casos, excepto en Maicao, se realizaron dos o tres módulos 1 de los cuales, los participantes eligieron sus representantes para el módulo 2. A su vez, del módulo 2 se escogieron los participantes del Módulo 3. Cada uno de los talleres tenía 35 personas como máximo. Para el Módulo 3 se invitaron decisores de las Secretarías de Salud, de las EPS-ARS y de las IPS, para que estuvieran presentes en la instalación de las Mesas de Trabajo. Después de evaluar cada taller se ajustaron las técnicas a cada uno de los contextos.

Diseño del Modelo de Interlocución

El Modelo de Interlocución se construyó a partir de una auto-reflexión sobre nuestro propio proceso metodológico, fundamentado en los pasos seguidos para identificar las necesidades de comunicación y definir las estrategias de intervención más adecuadas. Desarrollar un Modelo nos permitió precisar una metodología que identifica los actores locales, los problemas en la participación y en la comunicación, y las estrategias para resolver esos problemas.

\section{RESULTADOS}

Durante la primera y segunda fase se identificaron 1) los principales problemas funcionales y estructurales del régimen subsidiado, que llamamos grietas del sistema, así como los factores que afectan y que ocasionan estas grietas (Cuadro 1), para establecer las posibilidades de su mejoramiento en el nivel local y el margen de maniobra de los actores para incidir en el mismo; 2) identificamos la situación actual de la participación social, los espacios y mecanismos existentes, y el impacto de esta participación en el desempeño del sistema.

Además de la imposibilidad hasta el momento, de lograr la cobertura universal, se pudo constatar que el acceso y la calidad de los servicios se ven afectados por varios factores, pero principalmente por el incumplimiento o confusión de responsabilidades entre los diferentes actores institucionales. Por ejemplo, en el análisis del cumplimiento de las funciones por parte de empleados 
de las ARS y funcionarios de las Secretarías, se pudieron identificar las múltiples interpretaciones que estos hacen de la normatividad, las cuales se ven reflejadas en la prestación de los servicios. Por otro lado se estableció que los gobiernos locales están más enfocados a la ampliación de cobertura, descuidando la vigilancia del acceso y calidad de los servicios, y de la salud pública.

Cuadro 1. Factores y grietas del sistema de salud

\begin{tabular}{|c|c|c|}
\hline Factores & Grietas & $\begin{array}{c}\text { Principios y reglas } \\
\text { afectados }\end{array}$ \\
\hline $\begin{array}{l}\text { Inequidad entre los planes obligatorios } \\
\text { de salud POS } \\
\text { Población vulnerable mayoritaria } \\
\text { Retraso en la cobertura universal } \\
\text { Bajo desarrollo institucional del Estado y } \\
\text { pobre infraestructura para el manejo de } \\
\text { la información } \\
\text { Alta rolación de servidores públicos y de } \\
\text { empleados en las instituciones del } \\
\text { sistema } \\
\text { Conflictos de competencias y } \\
\text { responsabilidades } \\
\text { Brecha entre legisladores e } \\
\text { implementadores de las politicas } \\
\text { Intereses económicos por encima del } \\
\text { interés común de buena salud } \\
\text { Corrupción } \\
\text { Brecha de conocimiento entre los } \\
\text { actores del sistema } \\
\text { Factores de riesgo a la salud } \\
\text { (ambientales, de producción económica, } \\
\text { económicos, sociales, culturales, } \\
\text { políticos, estilos de vida), los perfiles } \\
\text { epidemiológicos } \\
\text { Tradición asistencialista en salud } \\
\text { Caracteristicas demográficas y } \\
\text { geográficas de la población local }\end{array}$ & $\begin{array}{l}\text { Delegación de la función pública a } \\
\text { entidades con intereses } \\
\text { económicos } \\
\text { Reformas y reglamentación } \\
\text { excesiva y frecuente } \\
\text { Desconocimiento por parte de } \\
\text { agentes de salud del } \\
\text { funcionamiento del sislema y de su } \\
\text { espíritu } \\
\text { Financiamiento del sistema } \\
\text { Fragmentación de las } \\
\text { responsabilidades y competencias } \\
\text { Desarticulación de la salud pública } \\
\text { y la salud individual } \\
\text { Politización de la salud } \\
\text { Interpretaciones erradas de las } \\
\text { normas } \\
\text { Débil vigilancia y control por parte } \\
\text { del Estado } \\
\text { Ciudadanos poco informados y } \\
\text { usuarios poco criticos } \\
\text { Condiciones sociales y políticas } \\
\text { desfavorables para la interlocución } \\
\text { Dificultades en el acceso a los } \\
\text { servicios } \\
\text { Baja calidad y oportunidad de los } \\
\text { servicios }\end{array}$ & $\begin{array}{l}\text { Principios: } \\
\text { Eficiencia } \\
\text { Universalidad } \\
\text { Solidaridad } \\
\text { Integralidad } \\
\text { Unidad } \\
\text { Participación } \\
\text { Reglas: } \\
\text { Equidad } \\
\text { Obligatoriedad } \\
\text { Protección integral } \\
\text { Libre escogencia } \\
\text { Autonomía de } \\
\text { instituciones } \\
\text { Descentralización } \\
\text { administrativa } \\
\text { Participación } \\
\text { social } \\
\text { Concertación } \\
\text { Calidad }\end{array}$ \\
\hline
\end{tabular}

Con relación a la participación pudimos determinar el campo relacional de los usuarios con el sistema, es decir los puntos de contacto, desde el proceso de identificación del beneficiario hasta la prestación de los servicios. A partir de dicho campo relacional, afinamos más los perfiles de los usuarios. Observamos los espacios de participación previstos por la ley: Atención al Usuario y alianzas de usuarios. Pero también otros espacios o personas, que facilitan o limitan la afiliación, el acceso y la oportunidad de los servicios: por ejemplo, empleados de EPS-ARS, del Sisben, de aseguramiento municipal, de la Caja y área de Trabajo Social de las ESE, de la coordinación de Atención de III y IV nivel y No POS de la Secretaría departamental de salud, entre otros. Estos puntos de contacto se activan principalmente cuando el usuario o alguien de su entorno está enfermo, o cuando le es negado un servicio. 
Varias son las interpretaciones locales de la participación. Los agentes institucionales se enfocan solamente en las capacitaciones programadas del usuario. En ocasiones se limita a estrategias de comunicación de las instituciones hacia sus usuarios. La participación también se asume como el cumplimiento de compromisos legales. Finalmente cuando existen los espacios de participación, estos no tienen resonancia en los agentes, pues prevalece el temor de estos a la crítica, por sobre la oportunidad de mejoramiento para la institución.

Desde la perspectiva del usuario-líder, no hay una comprensión de la utilidad y los beneficios de la participación ciudadana frente a su relación con el Estado. En La Guajira observamos que la participación es una oportunidad para constituir alianzas, principalmente políticas, con las instituciones. Frente a la carencia de fuentes de empleo el liderazgo se convierte en una posibilidad de movilidad social. Para el usuario no líder, la participación no está dentro de sus habilidades, por cuanto se siente imposibilitado por el desconocimiento y por su débil conciencia de derechos ciudadanos. El usuario en general relaciona los servicios subsidiados con la caridad o el asistencialismo.

Los problemas que los usuarios encuentran en el sistema, son resueltos a través de sus redes sociales y alianzas políticas. Algunos intentos organizativos se han visto truncados por la poca resonancia en las instituciones, generando frustración y desmotivación. En Riohacha mencionaron la resistencia institucional a proveer información a las veedurías, de manera que la Contraloría tuvo que tomar medidas coercitivas. En La Guajira se reveló el contexto político de los procesos organizativos. Algunos funcionarios mencionaron que en épocas electorales es peligroso realizar acciones colectivas pues pueden ser politizadas. Casi sin excepción, las alianzas de usuarios no propusieron una visión crítica de sus ARS. La única alianza visitada por nosotros y que tenía críticas fuertes hacia su entidad era la del Hospital de Riohacha.

Durante la fase III en la que se realizaron los talleres y se divulgaron los materiales educativos, la respuesta fue variada. Se evidenció que los procesos formativos y de capacitación toman tiempo, no solamente para la apropiación de conocimientos sino también con miras a un cambio de actitud frente al rol de ciudadanía. Si bien en algunos casos hubo un cambio notorio de la posición beligerante de exponer las quejas y buscar culpables, a la posición conciliadora y propositiva, buscando explicaciones y aportando a la solución, en otros casos, no fue fácil, transformar las actitudes tradicionales en los encuentros de ciudadanos con decisores, en el que la dinámica se caracteriza por un "pide-yda". Los talleres buscaban un cambio en los participantes, aportándoles 
herramientas y conocimientos para ser propositivos. Del lado de las instituciones, los decisores que tuvieron más contacto con el equipo de investigación y que se comprometieron desde un principio con el proyecto, fueron los más receptivos frente a las actividades propuestas y se comprometieron más claramente con la Mesa de Trabajo en Salud. Los materiales impresos, especialmente la Guía, tuvieron una muy buena recepción, hasta el punto que los usuarios-líderes la consultan cotidianamente. Se mostró como un importante instrumento de empoderamiento. Los usuarios con conocimientos básicos percibieron que los talleres tuvieron un mayor impacto y eran más útiles.

Durante la auto-reflexión del proceso investigativo, se definió el Modelo de Interlocución (Figura 1). En el Componente 1 "Conocimiento del contexto" se identifican los actores, los aspectos que permiten o no la interlocución (capacitación, fortalecimiento de la organización comunitaria, voluntad política, recursos, sensibilización de los agentes, diagnósticos) y las estrategias para crear un espacio de interlocución. En el Componente 2 "Preparación para la interlocución" se convocan e involucran los actores y se implementan las estrategias para garantizar la interlocución (talleres de capacitación, materiales pedagógicos, programas de radio y televisión, audiencias públicas, investigación, etc.). Durante el Componente 3 "Interlocución" se pone en marcha la Mesa de Trabajo en Salud, en donde se identifican y priorizan los problemas, se definen las causas, y se logran acuerdos frente a las soluciones, los plazos y los responsables.

Cuadro 1. Factores y grietas del sistema de salud

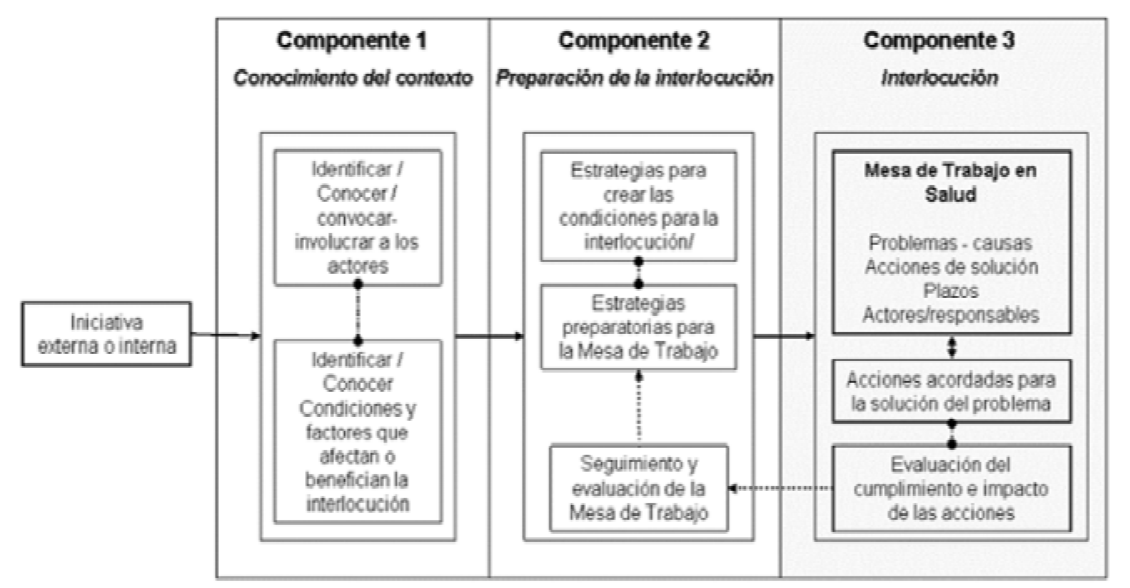


De este proceso, los resultados tangibles y que sirven como herramientas para replicar el Modelo de interlocución son los materiales educativos, que buscaron abordar el desconocimiento general que afecta la participación, y los módulos para la realización de talleres participativos. Los temas y el lenguaje utilizado responden a los distintos perfiles de usuarios. Los materiales podrían ser afinados y diversificados, utilizando por ejemplo, tecnologías de la información y comunicación TIC. Los talleres son útiles para reforzar el impacto de los materiales y para actualizar el conocimiento, pero también, permiten generar una base de multiplicadores que puedan replicar sus conocimientos en sus entornos comunitarios. Al final del tercer módulo los usuarios y agentes deben tener la actitud y las habilidades para dialogar en igualdad de condiciones, en un espacio de interlocución no beligerante que hemos llamado la Mesa de Trabajo en Salud.

\section{DISCUSIÓN}

Uno de los hallazgos más contundentes de la investigación fue el poco o nulo conocimiento de los usuarios sobre la estructura y funcionamiento del sistema, y los actores, responsabilidades y roles. Esto implica su incapacidad para ejercer derechos, la poca conciencia de su rol como ciudadanos y consumidores para exigir calidad, y la falta de recursos propios y motivación para incidir en el mejoramiento del sistema.

El Modelo de Interlocución es un conjunto de metodologías que propician las condiciones necesarias para la interlocución entre usuarios y decisores. A través del posicionamiento de todos los actores, principalmente de los usuarios, como interlocutores válidos y legítimos, este Modelo propicia la concertación de acciones de mejoramiento del sistema, mediante espacios y mecanismos permanentes y eficaces de diálogo.

El Modelo se ve materializado en la Mesa Local de Trabajo en Salud. Aunque en el sistema ya existen otros espacios y mecanismos de participación, nuestro objetivo no fue reemplazarlos, sino articularlos a un espacio de concertación más abierto, confiable y resultado de un trabajo conjunto. Hemos identificado de acuerdo a las circunstancias, a los actores y al contexto en el que se desee implementar el Modelo de Interlocución es necesario seguir los pasos que se muestran en la figura 1 , los cuales pueden variar según la información disponible, los recursos y los problemas que se deseen solucionar. 
El impacto de los talleres y materiales impresos fue en general positivo, gracias a que estaban basados en datos recogidos en campo, reflejando las necesidades y expectativas reales de los afiliados. La información recopilada en el diagnóstico institucional y en la etnografía, debe permitir establecer los mejores espacios y momentos para llevar a cabo las estrategias de intervención, los protocolos, rituales y códigos sociales para realizar la convocatoria y los talleres, y las formas de trabajo familiares para los participantes. En nuestro caso, faltó una mayor anticipación con base en la etnografía, en la escogencia de los horarios y lugares para realizar las actividades. Pero también es de especial cuidado la convocatoria a las instituciones. Un mayor esfuerzo en la sensibilización de los decisores es necesario para lograr su apoyo a las actividades del Modelo.

Las mayores dificultades para el diseño de los talleres fueron en la definición de actividades que transmitieran contenidos interesantes e inteligibles, que introdujeran claramente nuevos conceptos, incrementales en su complejidad, estructurados de una manera lógica (como si fuera un relato), permitiendo el un diálogo horizontal. Con los instrumentos para transmitir estos conocimientos y con el capital que aporta la etnografía y las técnicas participativas, se buscó que las dinámicas participativas tuvieran resonancia y motivaran. Después de las pruebas pilotos, comprendimos la necesidad de integrar un módulo adicional, dirigido a los agentes, para involucrarlos en el Modelo, sensibilizándolos e informándolos más sobre el sistema y sus metas, y sobre el rol de los usuarios en este proceso. Muchos de ellos conocen sus funciones y tareas, pero no tienen una visión completa del sistema, y menos aún, de dónde están ubicadas sus funciones. Tampoco están sensibilizados frente al espíritu del régimen subsidiado y a los derechos en salud.

En los talleres se buscó convertir al usuario en interlocutor. Se generó un ambiente no magistral, buscando un equilibrio entre la experiencia y conocimiento de los talleristas, y la experiencia y conocimiento de los asistentes. Se logró una mayor horizontalidad entre los actores en la Mesa, en los casos en los que los usuarios 1) se apropiaron de los conocimientos y léxicos, 2) valoraron sus propias experiencias, articulándolas de modo general y no personal, proponiendo soluciones, y 3) los agentes del sistema eran favorables a la interlocución.

Las estrategias permitieron en dos de los casos: 1) empoderar a los usuarios, convirtiéndolos en interlocutores, que por su conocimiento adquirido, son válidos y legítimos a los ojos de unos y otros; y 2) propiciar un espacio de confianza para la interlocución, como es la Mesa de Trabajo en Salud, en la cual la 
identificación de soluciones ganó más importancia que la problematización. En los otros dos casos, más trabajo con los usuarios y más sensibilización de las instituciones, son condiciones para el funcionamiento de las Mesas. En todos los casos, profundizar más en la formación de los agentes de salud, especialmente, el personal asistencial y agentes de primer contacto, evitaría su posición defensiva en la interlocución.

El gran riesgo de la participación es su tendencia a la politización, resultando en la pérdida de los intereses comunitarios o colectivos. Los usuarios perciben la participación como la intermediación en la resolución de problemas individuales o comunitarios, sin entender las posibilidades de la movilización colectiva para el mejoramiento de la salud de todos. Se observa que los procesos participativos son monopolizados por los líderes, fortaleciendo jerarquías y dependencias en las redes sociales y políticas. Sin embargo, con la capacitación de la base comunitaria, se logró, en algunos de los casos, que el líder convirtiera su conocimiento en acciones propositivas.

Observamos que las Mesas de Trabajo en Salud que permanecieron en funcionamiento se caracterizaban por: 1) la convocatoria exitosa de por lo menos la secretaría de salud y el hospital local; 2) la apropiación por parte de los usuarios de la metodología de concertación y diálogo de la Mesa; 3) el compromiso de todos los actores para negociar soluciones y cumplir con los acuerdos; y 4) mecanismos de retroalimentación con las comunidades representadas por los usuarios.

Para dejar un proceso más fortalecido es necesario más tiempo de preparación y de creación de la Mesa, por dos motivos: 1) mejor apropiación de la metodología de trabajo y mejor comprensión de la finalidad y posibilidades de la Mesa; 2) mayor convocatoria a las instituciones del sector, quienes se desinteresaron de la Mesa por la rotación y discontinuidad en los agentes institucionales, la poca comprensión sobre su utilidad y por el esfuerzo institucional que implica un proceso participativo.

No obstante la literatura y los documentos oficiales que recomiendan la participación, cabe la pregunta por el beneficio que trae la participación ciudadana para el Estado, las ARS y las IPS. Creemos que los beneficios para los usuarios son muchos, pero no es tan obvio para las instituciones. Por lo menos dos pueden mencionarse: 1) la interlocución facilita el mejoramiento de la prestación, la continuidad y la racionalización de los recursos invertidos, respondiendo mejor a necesidades y expectativas; 2) el apoyo a las acciones y 
políticas de las entidades cuando estas son concertadas con la población. Se debe tener en cuenta para convocarlos, que su participación va ligada a su percepción de los beneficios posibles.

Según nuestras observaciones en La Guajira, independientemente del interés que tengan las ARS y las IPS, por lo menos dos de las Mesas están en capacidad de instarlas a participar, siempre y cuando sigan contando con el apoyo de la administración municipal. Participar en la Mesa, por ejemplo, les permitiría mejorar su imagen frente a las críticas actuales a su desempeño. De hecho, en algún momento, los miembros de las Mesas plantearon la posibilidad de asumir como criterio de escogencia de una ARS su participación en la Mesa de Trabajo. Para que el Modelo pueda ser replicado es esencial, por lo menos, el apoyo del gobierno local. En dos de nuestros casos, las secretarías de salud y las EPSARS mostraron poco interés. En cambio, los hospitales estatales estuvieron más prestos a participar, sensibles a la necesidad de lograr el apoyo de los usuarios. Por ello, la convocatoria institucional debe ser más contundente.

De acuerdo al desarrollo preliminar del Modelo consideramos unos componentes que deben ser previos a la convocatoria e instalación de la Mesa de Trabajo, pero es posible que al desarrollarlo más, se encuentren "atajos metodológicos" y adaptaciones a los diferentes contextos locales del régimen subsidiado. El propósito del Modelo es ofrecer unos lineamientos de acción para iniciativas que busquen mejorar la participación social y lograr mayor incidencia de la población en los procesos de planificación de salud. Por ello, el conocimiento del contexto y de los actores es fundamental para diseñar las estrategias que redunden en la interlocución. Pero la Mesa de Trabajo en Salud se reveló pertinente, pues mediante los acuerdos logrados con los decisores, se logra la incidencia real del usuario en el desempeño local del sistema de salud, fin principal de este Modelo •

Agradecimientos. Este artículo es un resultado del proyecto "Representación de las comunidades locales en el régimen subsidiado de salud: el caso del Departamento de La Guajira", realizado por el grupo Recursos Estratégicos, Región y Dinámicas Socioambientales RERDSA (clasificado B) del Instituto de Estudios regionales INER de la Universidad de Antioquia. Este proyecto se co-financió entre Colciencias y la Universidad de Antioquia, y participaron la Secretaría Departamental de Salud de La Guajira, la Universidad de La Guajira, la ESE Hospital Indígena de Nazareth y la Asociación Colombiana de Salud, ASSALUD. Agradecemos a todos los miembros del equipo de investigación, pues algunas de las consideraciones aquí expresadas son resultado de discusiones colectivas. Nuestra gratitud va dirigida especialmente, 
a las comunidades guajiras por estar siempre prestas a participar de nuestros proyectos, los cuales han buscado contribuir al mejoramiento de sus condiciones de vida, pero ninguno como éste, había logrado interpelar su interés y su capacidad para continuar con los procesos iniciados.

\section{REFERENCIAS}

1. Organización Mundial de la Salud OMS. Informe sobre la Salud en el Mundo 2000. Mejorar el desempeño de los sistemas de salud. Ginebra: OMS; 2000.

2. Mosquera M, Zapata Y, Lee K, Arango C, Varela A. Strengthening user participation through health sector reform in Colombia: a study of institutional change and social representation. Health Policy and Planning 2001; 16(2): 52-60.

3. Restrepo H. [Internet]. Incremento de la capacidad comunitaria y del empoderamiento de las comunidades para promover la salud. Quinta Conferencia Mundial de Promoción de la Salud México, D.F. 5 al 9 de junio de 2000 Disponible en: http:/ /www.paho.org/Spanish/AD/SDE/HS/5thGlobalConfSp6.doc. Consultado Noviembre de 2006.

4. Deppe HU. [Internet]. The present situation and perspectives of universal systems of health. Ponencia 11th World Congress on Public Health, Agosto 21-25 de 2006, Rio de Janeiro. Disponible en: http://www.ensp.fiocruz.br/parcerias/redsalud/ bibliografias/Rio06b.PDF. Consultado en Noviembre de 2006.

5. Rodríguez-Monguió R, Infante Campos A. Universal health care for Colombians 10 years after Law 100: challenges and opportunities. Health Policy 2004; 68: 129142.

6. Calvelo JM. [Internet]. Los Modelos de Información y de Comunicación. El Modelo de Interlocución: un Nuevo Paradigma de Comunicación. FAO 1998. Disponible en: http://www.fao.org/sd/SPdirect/CDan0022.htm. Consultado Noviembre 2006.

7. Winkin, Y. Anthropologie de la communication. De la théorie au terrain. Bruselas: De Boeck Université; 1996.

8. Rizo García M. [Internet]. El Camino Hacia la "Nueva Comunicación". Breve Apunte Sobre las Aportaciones de la Escuela de Palo Alto. Razón y Palabra 2004; 40. Disponible en: http://www.cem.itesm.mx/dacs/ publicaciones/logos/anteriores/n40/mrizo.html. Consultado Mayo 2007.

9. Hubley J. [Internet]. Health Empowerment, Health Literacy and Health promotion - putting it all together. Disponible en: http://www.hubley.co.uk/ 1hlthempow.htm. Consultado Noviembre de 2006.

10. Kickbusch I, Wait S, Maag D. [Internet]. Navigating health. The role of health literacy. Alliance for Health and the Future, International Longevity Centre-UK. Disponible en: http:/www.healthandfuture.org/publications/ issue_briefs/pdf/Navi gatingHealth.pdf. Consultado Noviembre 2006. 
11. Pitta, A. M. R.; Rivera, F. J. U. Sobre pontos de partida: planejamento em comunicação e integralidade da atenção em saúde. Interface - Comunic., Saúde, Educ. 2006; 10 (20): 395-410.

12. McGregor, J. Allister. Researching Well-Being. Communicating between the Needs of Policy Makers and the Needs of People. Global Social Policy 2004; 4 (3): 337-358.

13. Delgado-Gallego ME, Vázquez-Navarrete L. Barreras y Oportunidades para la Participación Social en Salud en Colombia: Percepciones de los Actores Principales. Rev Salud Pública-Colombia 2006; 8 (2): 128-140.

14. Puerta C. Roles y estrategias de los gobiernos indígenas en el sistema de salud colombiano. Revista Colombiana de Antropología 2004; 40: 85121. 